\title{
Über das Faxen in den Praxen
}

\section{Eberhard Wolff}

PD Dr. rer. soc., Redaktor Kultur, Geschichte, Gesellschaft

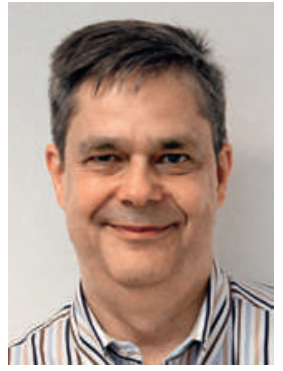

Neulich musste ich ein Dokument zügig an einen älteren Herrn in einer Seniorenresidenz übermitteln. Per Internet ging es gerade nicht. Der Postweg war zu langsam. Da meinte der Herr, ich solle doch einfach einen Fax schicken.

Einen Fax?!?!?!? Diese «Steinzeit-Technologie» ist seit etwa zehn Jahren aus meinem privaten und beruflichen Umfeld verschwunden. Selbst die Senioren(!)-Residenz gab im Internet keine Faxnummer mehr an.

Totgesagte leben bekanntlich länger [1]. Auch und gerade in Arztpraxen. Aus der «SISA II»-Umfrage [2] können wir schliessen, dass das Faxen in den meisten Schweizer Arztpraxen immer noch eine Selbstverständlichkeit ist [3], selbst wenn dort ein Praxisinformationssystem läuft. $69 \%$ der End-Validierungen der «medica»-Labore z.B. kommen auch oder nur per Fax in die Praxen.

eHealth-Verfechter sehen darin ein Defizit und wollen dies ändern. Die voll digitalisierte und vernetzte Arztpraxis dräut am Horizont. Ich will die Sache hier umkehren und fragen: Warum glühen die Faxgeräte in den Arztpraxen (noch)?

Klar: Wenn der Befund des kleinen Labors hereinkommt oder das dringende Rezept an die kleine Apotheke herausgeht, also die digitale Schnittstelle fehlt, muss selbst die modernste Arztpraxis via Fax kommunizieren. Dies wird mit der Zeit aber seltener werden.

Manche Praxen mögen ihre Vorgänge aus Gewohnheit mit dem Faxgerät abwickeln. Oder sie trauen dem Gerät eher zu, dass die Informationen sicher beim Adressatenund insbesondere nur dort - ankommen. Das Faxgerät ist für sie der «Dieselmotor» der Praxiskommunikation. Vor allem aber kann das materielle Faxen in speziellen Situationen praktischen Sinn machen. Mir wurde nicht nur von einer Praxis erzählt, in der die papiernen Laborwertfaxe einen effizient organisierten Parcours durchlaufen. Das Papier macht schon bei der Ankunft auf sich aufmerksam (manchmal werden nur die pathologischen Werte gefaxt), es ist einfach im Blick zu behalten. Je nach Dringlichkeit wandert es in bestimmte Ablagen, zu bestimmten Mitarbeitenden. Der Überblick über die Pendenzen bleibt gewahrt, der Medienkanal ist überschaubar, nicht verzweigt. Man kann den Fax an jeden Ort mitnehmen, nichts muss am nächsten Rechner neu aufgestartet werden. Und kein Problem, wenn der Kaffee darauf ausleert. Der Fax garantiert in diesem Sinne Praxisqualität, entstanden aus praktischer Vernunft vor Ort.
Auch der Baubereich liebt offenbar das Faxen. Vielleicht ist der Fax gerade in den Branchen so lebendig, in denen körperlich an Mensch oder Material und an vielen Orten gearbeitet wird.

Genau genommen wäre es für mich selber auch einfacher, wenn ich die handschriftlich korrigierten Satzabzüge meiner SÄZ-Artikel nicht nach Muttenz mailen, sondern nur ins Faxgerät legen könnte. Das machen einige (noch) so, sagt unsere Managing Editorin Tanja Kühnle. Danke, Tanja, Du machst mich so unwillentlich vielleicht noch zum Neofaxer.

Und Sie? Falls Sie diesen Artikel zufällig als Print in Händen halten: Schätzen Sie auch die anschmeichelnde Ästhetik des Anfassens und Umblätterns, den Geruch von Druckfrischem, den leichten Schimmer des gestrichenen Papiers?

Zwei von drei "medica»-Laborwert-Faxen kommen ganz findig zusätzlich zum E-Datenstrom. Ich denke, dass eine gewöhnliche Arztpraxis ständig auf mindestens fünf verschiedenen Kommunikationskanälen arbeitet. «Patchwork»-Arbeitsweisen [2] nennen das die eHealth-Forschenden. Sie betrachten dies als ineffiziente Arbeitsweise, die es zu überwinden gilt. Die Idee der «papierlosen Praxis» erscheint als eine Art Wiedergänger: Als die Computer Laufen lernten, entstand die Fantasie eines "papierlosen Büros». Wir wissen, was daraus wurde.

Jenseits der eHealth-Perspektive ist «Patchwork»-Kommunikation aber der Normalfall, in der Arztpraxis ebenso wie im sonstigen Leben. Der zahlenmässige Effizienzgewinn des digitalen Datenflusses ist nicht der einzige Aspekt einer guten Praxiskommunikation. Ein kleines Quiz: Welche Wege boten die FMH-Services im letzten Jahr für die Anmeldung zum «Praxiscomputer-Workshop» (!) in der SÄZ an? Die Antwort lautet: Homepage (erst von dort aus zum Webformular), Fax und Telefon, gleichwertig nebeneinander stehend. Das ist für mich in diesem Sinne nicht inkonsequent, sondern sympathisch und realistisch, weil es PatchworkKommunikation als Normalität akzeptiert.

Übrigens: Meinen Fax ins Seniorenheim konnte ich schliesslich noch abschicken: mit einem Internet-FaxService. Das Heim hatte nämlich unter der Hand doch noch ein Gerät am Laufen. Ich habe es über eine alte, ungelöschte Website herausgefunden.

Ich wette: Irgendein «Patchwork» wird immer überleben. Wenn nicht offen, dann heimlich. 